\title{
Ion Beam Stimulation Therapy With a Nanoradiator as a Site-Specific Prodrug
}

\author{
Jong-Ki Kim ${ }^{1 *}$, Seung-Jun Seo ${ }^{1}$ and Jae-Geun Jeon ${ }^{2}$ \\ ${ }^{1}$ Department of Biomedical Engineering and Radiology, School of Medicine, Daegu Catholic University, Daegu, South Korea, \\ ${ }^{2}$ Department of Periodontology, School of Dentistry, Kyungpook National University, Daegu, South Korea
}

\section{OPEN ACCESS}

Edited by:

Marco Durante,

GSI Helmholtz Center for Heavy lon

Research, Germany

Reviewed by:

Narayan Sahoo,

University of Texas MD Anderson

Cancer Center, United States

Giacomo Cuttone

Laboratori Nazionali del Sud

(INFN), Italy

Yoshitaka Matsumoto,

University of Tsukuba, Japan

${ }^{*}$ Correspondence:

Jong-Ki Kim

jkkim@cu.ac.kr

Specialty section:

This article was submitted to

Medical Physics and Imaging,

a section of the journal

Frontiers in Physics

Received: 27 April 2020

Accepted: 17 June 2020

Published: 31 August 2020

Citation:

Kim J-K, Seo S-J and Jeon J-G (2020) Ion Beam Stimulation Therapy With a Nanoradiator as a Site-Specific

Prodrug. Front. Phys. 8:270.

doi: $10.3389 /$ fphy. 2020.00270
In view of the fact that Bragg peak energy cannot be delivered individually to multiple scattered infiltrating tumors or diffuse lesions, the energy of the ion beam could instead be adjusted to traverse the entire body for the selective activation of nanoparticles (NPs) inside the target lesions with an ion fluence comparable to the Bragg peak. This Coulomb stimulation of NPs generates low-energy electrons (LEES) and characteristic fluorescent $X$-rays (XFLs) from the NP surface; this effectively transforms inert NPs into nanoradiators, much like the conversion of a prodrug into a drug. In contrast, the relatively small plateau dose absorbed along the beam path ensures that there are minimal effects to normal tissue (NT). This simple but innovative approach enables unprecedented traversing ion beam stimulation therapy (TIBS) for infiltrating tumors or diffuse non-oncological lesions. The theoretical background and efficacy of TIBS has been demonstrated by several proof-of-concept studies with animal disease models and molecular-targeted high-Z NPs.

Keywords: ion transmission beam, Coulomb stimulation, high-Z nanoparticles, site-specific dose enhancement, therapeutic beacon, diffuse lesions, molecular targeting

\section{INTRODUCTION}

A proton or carbon ion beam dissipates energy at a specific depth by forming a Bragg peak during its passage through tissue depending on its energy; this concept has been used to deliver a therapeutic radiation dose to the target in conventional proton or carbon ion therapy. In contrast, traversing ion beam stimulation therapy (TIBS) is performed by a traversing ion beam, whose energy reaches beyond the depth at which a Bragg peak forms in the tissue, to activate high- $\mathrm{Z}$ nanoparticles (NPs) that can be either delivered to the pathological lesions by various targeting schemes or intrinsically integrated into the target by a pathological process. The ion fluence decreases gradually during this traversal due to nuclear reaction-mediated ion loss and abruptly reduces to zero at $\delta$ while being absorbed in medium with a liberating Bragg peak dose (BPD) $[1,2]$. Consequently, the ion fluence passing through the NP-containing target is markedly larger than the number of ions absorbed in the surrounding NT, which can only reach the plateau dose (PD), as shown in Figure 1. Traversing ions selectively facilitate physical Coulomb interactions with high- $\mathrm{Z} \mathrm{NPs} \mathrm{(Pt,} \mathrm{Au}, \mathrm{Gd}$, and $\mathrm{Fe}$ ) in the target tissue, which is achieved by a relatively higher $\mathrm{Z}$ number and a higher ion-impact dose on NPs than on tissue elements. TIBS ionizes atoms via inelastic Coulomb scattering with atomic electrons in high-Z NPs, and as they pass, the NPs emit low-energy electrons (LEEs) and fluorescent X-rays (XFLs) via both Auger cascades [3,4] and interatomic Coulomb decay (ICD) during the de-excitation process [5, 6], termed the Coulomb nanoradiator effect (CNR) [7]. 


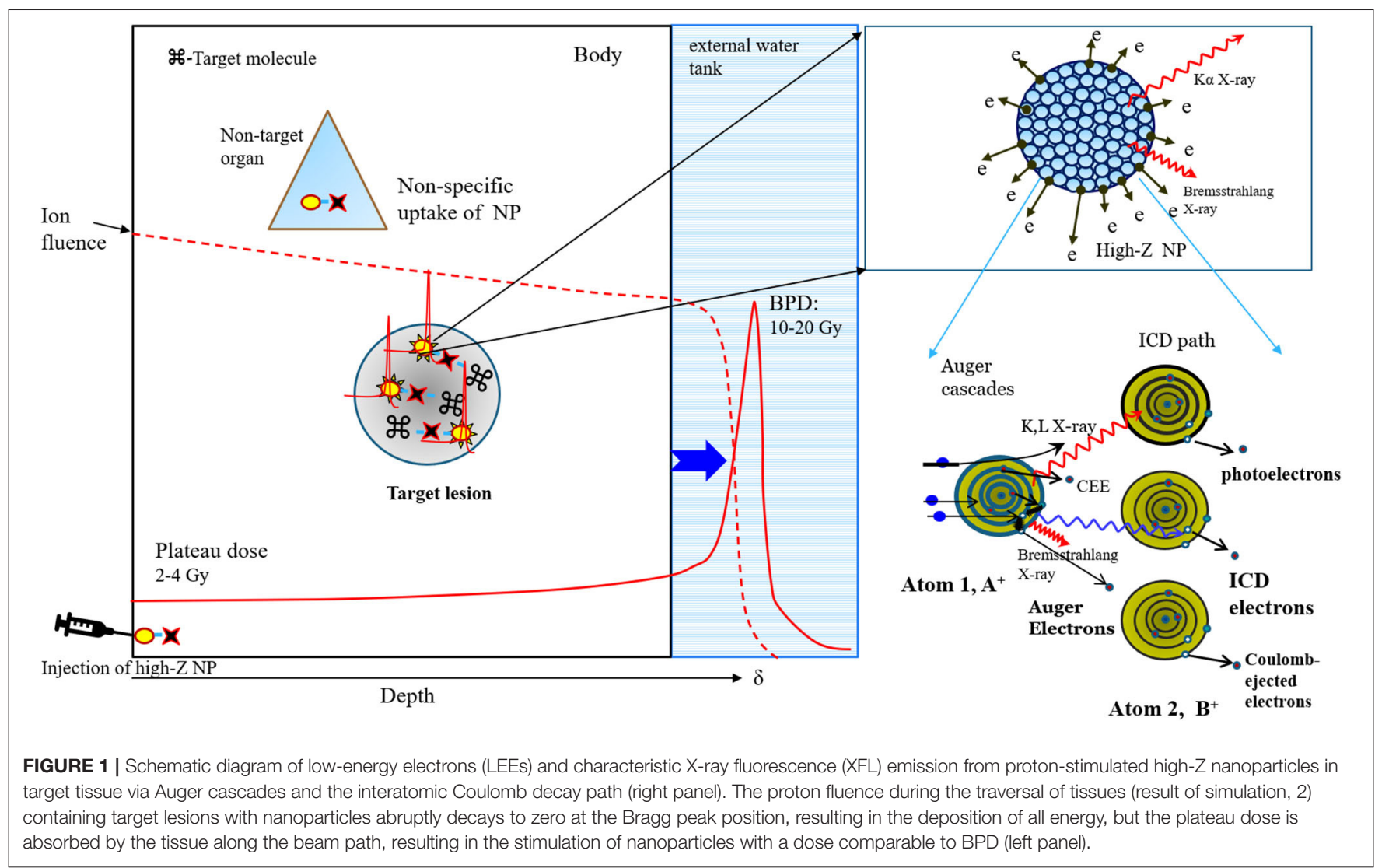

CNR-derived LEEs break up bonding of adjacent biomolecules in the target lesions directly $[8,9]$, resulting in damage to the lesions. Diffuse and multiple scattered lesions in various intractable cancers and non-oncological conditions, such as neoangiogenesis, atherosclerotic plaques with thrombosis, and neurodegenerative plaques, are excluded from the indications for radiotherapy or surgical treatment. Previously, bare ironoxide nanoparticles were used for the treatment of either arterial thrombosis or brain glioma. Here, we designed new nanoparticles to target either LDL receptors for $\mathrm{BBB}$ crossing and glioma or scavenger receptors of macrophages and active thrombus in atheroma for better targeted delivery and site-specific activation by TIBS. In this work, we present the theoretical background for precise and effective therapeutic proton-TIBS with moleculartargeted high-Z NPs in animal disease models.

\section{MATERIALS AND METHODS}

\section{Synthesis of Molecular-Targeted High-Z Nanoparticles}

LDLR-targeting ApoB@AuNPs-Cy5 were synthesized by thiolating the ApoB peptide (3-mercaptopropionic acidDWLKAFYDKVAEKLKEAFRLTRKRGLKLA-NH2: Anygen, Korea) and SH-PEG5000-Cy5.5 (Intechim, France) to $15 \mathrm{~nm}$ gold NPs (Nanoprobe, USA) using the same method described previously [10]. Scavenger receptor-A1 (SR-A1)-targeting
PP1@AuNPs-Cy5 or VEGFR-targeting Flt1@AuNPs-Cy5 were also synthesized by thiolating the PP1 peptide (3mercaptopropionic acid-LSLERFLRCWSDAPA-NH2) or the Flt1 peptide (3-mercaptopropionic acid-GGNQWFI-NH2) and SH-PEG5000-Cy5.5 to $15 \mathrm{~nm}$ AuNPs in the same way described above. Briefly, $1 \mathrm{~mL}$ stock solution of citrated AuNPs $(0.5 \mathrm{mg} / \mathrm{mL}, 2.538 \mathrm{mM})$, SH-PEG-Cy5.5 (5 mg/mL, $1 \mathrm{mM})$ in distilled water, and either Flt1 or PP1 peptide $(2.6 \mathrm{mg} / \mathrm{mL}$, $2.863 \mathrm{mM}$ ) in dimethyl sulfoxide (DMSO) were prepared. In a typical synthesis of $10 \mu \mathrm{M}$ Flt1@AuNP-Cy5.5 or PP1@AuNPCy5.5 nanocomplexes, $928.56 \mu \mathrm{L}$ of distilled water was added to a bottle prior to mixing with $50 \mu \mathrm{L}$ of SH-PEG-Cy5.5 and 17.5 $\mu \mathrm{L}$ of $100 \mu \mathrm{M}$ each peptide for $5 \mathrm{~min}$ while stirring to produce a 1:1 molar ratio of SH-PEG-Cy5.5 to the peptide. Then, 3.94 $\mu \mathrm{L}$ of AuNP solution was added to the mixture of SHPEGCy5.5/Flt1 or PP1 peptide, resulting in a 5:1 molar ratio of thiol to gold at $\mathrm{pH}$ 7.4. Finally, the mixture of SH-PEG-Cy5.5/Flt1 or $\mathrm{PP} 1 / \mathrm{AuNPs}$ was stirred at room temperature for $2 \mathrm{~h}$ to allow for the complete formation of gold nanocomplexes. To purify the complex, reactants were centrifuged at $16,000 \mathrm{rpm}$ for $10 \mathrm{~min}$. After decanting the supernatant, the pellet was resuspended in 0.5 or $1 \mathrm{~mL}$ of phosphate-buffered saline (PBS).

Thrombus-targeting Fuc@Fe3O4 NPs were synthesized by conjugating aminated fucoidan to citrated iron oxide NPs (ION) using EDC/NHS chemistry. Aminated fucoidan was prepared by reacting epichlorohydrin-treated fucoidan with $30 \%$ ammonia 
water at $40^{\circ} \mathrm{C}$ for $90 \mathrm{~min}$ [11]. Briefly, we prepared $10 \mathrm{ml}$ of ION solution by adding $4 \mathrm{ml}$ citrated ION solution $(40 \mathrm{mg})$ to $6 \mathrm{ml}$ MES buffer ( $\mathrm{pH} 6$ ). A total of $1-10 \mathrm{mg}$ of aminated fucoidan was dissolved in $1 \mathrm{ml}$ PBS buffer. We then added $0.4 \mathrm{mg}$ EDC to the ION solution and stirred for $15 \mathrm{~min}$ after adding $1.1 \mathrm{mg}$ sulfo-NHS to ION solution and adjusting $\mathrm{pH}$ to 7.0 using sodium bicarbonate $(0.7 \mathrm{ml})$. Then $1 \mathrm{ml}$ of aminated fucoidan was mixed with ION solution containing EDC/NHS and stirred for $2 \mathrm{~h}$. Fucoidan-conjugated ION was condensed with strong magnet to separate unreacted components which was decanted finally after washing PBS several times. The formation of peptide bonding was confirmed by FT-IR spectroscopy.

\section{Animal Models}

The procedures used for the laboratory animals were approved by the Institutional Animal Care and Use Committee of Catholic University Hospital of Daegu (approval numbers, DCIAFCR 151007-7-Y and 181029-22Y).

\section{F98 Rat Glioma Model}

A total of $2 \times 10^{5}$ F98 glioma cells were implanted in the frontal lobe of Fischer 333 rats by stereotactic surgery as described previously [12]. Briefly, after immobilizing the rats in a rodent stereotactic frame, an incision was made in the skin, and a burr hole was made in the skull. One million tumor cells were injected at a rate of 1-2 microliters/minute using a microsyringe (Hamilton, Reno, NV, US) mounted on a stereotactic frame (Kopf Instruments, Tujunga, CA, US) at coordinates of $1 \mathrm{~mm}$ lateral and $1 \mathrm{~mm}$ posterior to the bregma and $1.5 \mathrm{~mm}$ below the dura. Tumor growth was evaluated with $4.7 \mathrm{~T}$ MRI 5 days after implanting the cells. In this study, a total of six rats were divided into two experimental groups for two irradiation doses ( 10 and $5 \mathrm{~Gy}$ ) and three rats were left untreated to act as a proton alone control.

\section{Ligated-Artery Mouse Atheroma Model}

A partial ligation of the left coronary artery (LCA) was carried out in C57BL/6 mice $(n=6)$ as previously described [13]. Briefly, three of four caudal branches of the LCA (left external carotid, internal carotid, and occipital artery) were ligated with 6-0 silk sutures, while the superior thyroid artery was left intact. C57Bl/6 mice were continuously fed a high-fat diet post-ligation. After 4 weeks, the mice were monitored by blood flow measurement and surgery to identify atheromatous plaque in the affected vessel. Three mice were treated with TIBS, and the other three were untreated as control.

\section{Proton-TIBS}

Proton irradiation was performed at KOMAC TR102 (Kyungju, South Korea). Intraperitoneal injections of $100 \mathrm{mg} / \mathrm{kg}$ ketamine and $20 \mathrm{mg} / \mathrm{kg}$ xylazine cocktail were administered under anesthesia. A pristine proton transmission beam, with the Braggpeak behind the body (PS), was irradiated at $24 \mathrm{~h}$ after injection of NP, based on results of previous measurement of maximum uptake in tumor [7] or thrombus [14].

The rat or mouse was positioned in an upright position by tying a thread around a tooth in a sample mounter. Then the proton beam traversed through the head of the rat or the neck of the mouse from anterior to posterior. Rats that had grown tumors and mice that had developed obstructed arteries with atheroma were irradiated using a designed collimator and blocks to avoid unnecessary exposure to radiation. The irradiation energy was $100 \mathrm{MeV}$, which is sufficiently high to traverse the head of the rat or the neck of the mouse, and the single entrance dose was either 10 or $4 \mathrm{~Gy}$, as measured by a TM30013 Farmer chamber (PTW), at the frontal surface of the sample.

\section{Histologic and Imaging Analysis}

The flow patency of the ligated atheroma mouse model was evaluated using a Doppler flow meter 7 days after treatment, and the mice were then euthanized. The treated vessels were removed and then subjected to fixation and staining for histologic analysis. The areas of the remaining thrombus from three different cross-sectional planes (middle, proximal, and distal; thickness of $20 \mu \mathrm{m}$ ) in the dissected vessels were measured using default image analysis software with an optical microscope (Axiophot, Zeiss, Germany).

Glioma model rats were killed 7 days after treatment by an overdose injection of sodium pentobarbital. The brains were removed, fixed in $10 \%$ formaldehyde, paraffin embedded, and sectioned through the area of irradiation. The $5-\mu \mathrm{m}-$ thick sections were stained with hematoxylin and eosin, and the tumor was examined microscopically. Fluorescence imaging of extracted brain hemispheres was performed $24 \mathrm{~h}$ after IV injection of ApoB@AuNPs-Cy5 in F98 rat glioma models using an in vivo fluorescence imaging system (FOBI, NeoScience Co. Ltd, Korea).

Human retinal microvascular endothelial cells (HRMECs) treated with Flt1@AuNPs-Cy5 were embedded in two-chamber slides (SPL, Korea) using a mounting medium (Dako, Denmark) and examined using an optical microscope or a fluorescence confocal microscope (Nikon confocal microscope A1, Japan).

\section{RESULTS AND DISCUSSION}

\section{Theoretical Background of TIBS}

\section{Therapeutic Beacon of High-Z NPs With CNR}

Prior to becoming nanoradiators under TIBS, NPs retain inert prodrug status without cytotoxic effects due to appropriate coating $[7,15,16]$. Therefore, even if non-specific uptake by nontarget organs or tissues occurs, the NPs do not exert harmful effects as long as the ion beam is not irradiated incidentally on the area of non-specific NP absorption. Since the locations of lesions associated with cancer or other diseases are normally identified by clinical imaging studies prior to therapy, ion beams can be irradiated specifically to target lesions, generating virtual drugs from high-Z NPs with CNR effects while avoiding the exposure of non-targets. This advantage may resolve the major bottleneck in nanomedicine by avoiding side effects from non-specific uptake of nanoforms of drugs by unwanted organs or tissues, which occurs often after administration, particularly via intravenous injection. Non-specific delivery of NPs is unavoidable and occurs irrespective of conjugating the NPs to targeting marker molecules in the circulatory system [17]. 
One way to overcome this problem is to design cleavable molecular links between nano-formulated drugs and inhibitor molecules that are cleaved by tissue-specific enzymes overexpressed in the target lesion [18]. Cleavage of the linking molecule by the enzyme liberates the inhibitor and produces the drug effect of the nanodrug. However, this method is limited due to the lack of such lesion/tissue-specific cleaving enzymes. In contrast, TIBS can activate the drug effect from any high-Z NPs delivered to the target site, irrespective of the biochemical status of the lesion.

\section{Comparison of Traversing Ion Beams With X-Ray Photons}

\section{Depth-dose distribution}

Both ion beams and X-ray photons can activate high-Z NPs but have different interaction mechanisms and depth-dose distributions when passing through the tissue. Conventional broad-band X-rays with an energy of $\mathrm{MeV}$ yield much larger entrance doses than traversing pristine ion beams; thus, X-ray irradiation should be spatially and temporally fractionated to activate NPs safely by photoelectric absorption. Monochromatic $\mathrm{X}$-ray photons such as 50 or $68-82 \mathrm{KeV}$ photons that excite either Gd or Au NPs show a depth-dose distribution comparable to that of traversing pristine ion beams, but intense beams are only currently available in synchrotron radiation facilities. Traversing pristine ion beams only deposit PDs while passing through the body via multiple Coulomb scattering (MCS), so the majority of the ion fluence is transmitted to and interacts with high-Z NPs by Coulomb scattering, as depicted in Figure 1. Importantly, this property of the traversing ion beam enables selective activation of the high-Z NP prodrug while preserving the surrounding NT, and emerging TIBS is feasible for various diseases that were formerly not indicated for radiotherapy or surgical intervention.

\section{Interaction with high-Z NPs}

When an ion beam impacts NPs, it first ionizes compositional atoms of the NPs by inelastic Coulomb scattering, and it then yields CNR effects on both ionized and neutral atoms during the deactivation process. Ionization may occur simultaneously at multiple electronic levels, yielding CNR in multiple levels of a given impact atom and surrounding neutral atoms. X-ray photons ionize the NP atoms mainly through photoelectric absorption, which produces similar nanoradiator effects during deactivation. Monochromatic X-rays induce a nanoradiator effect in resonance on the K-line electrons, while broad-band X-rays potentially induce multiple energy levels through additional Compton scattering but are subject to attenuation and elevated entrance doses. Consistently, a simulation study demonstrated that a monochromatic $\mathrm{Au}$ $\mathrm{K}$-line X-ray was more effective in inducing stronger dose enhancement than a $\mathrm{MeV} \mathrm{X}$-ray under a given irradiation dose [19].

Under an entrance dose of $10 \mathrm{~Gy}$ and the same dose of Gdoxide NPs, the relative nanoradiator dose from irradiation with a $45 \mathrm{MeV}$ proton stimulation was compared to that from a $50 \mathrm{KeV}$ synchrotron X-ray photon. The proton-derived enhancement was 1.58 times larger than the X-ray-derived enhancement [20].

We tried to compare the therapeutic efficacy of $\mathrm{Au}$ nanoradiators in proton stimulation therapy in a Balb/c tumor model with that of Au-nanoradiators in broadband X-ray irradiation using gold NPs of the same size for a similar tumor size. Complete tumor regression was achieved either by X-ray-derived photoelectric nanoradiators with tumor uptake of $3.2 \mathrm{mg} \mathrm{Au/g}$ tissue or by protonstimulated nanoradiators with 58-79 $\mu \mathrm{g}$ Au/g tissue in tumors [7, 21]. This suggests that the proton-TIBS is more efficient for producing the same therapeutic effect with a relatively smaller tissue density of AuNPs. Taken together, these results show that producing the nanoradiator effect by Coulomb scattering on multiple inner valence levels or on multiple electrons in the inner shell of an NP atomic cluster from high-energy proton-TIBS has received relatively higher rates of induction compared with X-ray photoelectric nanoradiators based on the resonance of energy matched to a valence level.

In addition, iron oxide NPs can be effectively activated to produce CNR effects by high-energy protons or carbon ion beams, but neither monochromatic $7 \mathrm{keV}$ X-rays (corresponding to Fe-K lines) nor $\mathrm{MeV} \mathrm{X}$-rays $[19,22]$ could produce nanoradiator doses effectively due to either rapid attenuation in deep tissue or inefficient photoelectric absorption and production of Auger and ICD-type electrons from Fe atomic clusters, respectively. However, combining $7 \mathrm{keV} \mathrm{X}$-ray and iron oxide NPs is an effective tool in treating superficial lesions, such as a recurrence of breast cancer on the chest wall or cutaneous lymphomas [23]. Therefore, compared with X-ray photons, TIBS may exploit NPs with a relatively wider range of Zvalues to generate nanoradiator doses. Dose-enhancement from the proton-mediated CNR effect was previously reported [24].

\section{Comparison of Traversing Ion Beams With Neutron Beams in BNCT}

There was no direct quantitative comparison study between TIBS and BNCT using the same animal model. Neutron beams have a similar depth-dose distribution, including escalation of the entrance dose, with the broad band X-ray photon. Boron compounds in the target can capture neutron beams as X-rays are photoelectrically absorbed in high-Z NPs to enable them to emit electrons. Therefore, the depth-dose is different to that of the ion transmission beam with the Bragg-peak behind the body in which transmission ion fluence is comparable to Bragg-peak and may bring about fundamentally different efficiency and mechanism in interaction with receiving NP to generate dose enhancement. To compare therapeutic efficacy between TIBS and BNCT, it is necessary to prepare the same size of an NP sample in the same disease model under the same irradiation dose. This study will be performed in collaboration with a Japanese group in the near future.

In general, an ion beam is efficient and convenient at delivering to the target due to the charged particles being 
comparable with neutron particles. Moreover, preparation for molecular targeting of high-Z NP in TIBS is relatively wellestablished compared with targeting a boron compound in BNCT. However, a specific molecular probe is required for each disease in TIBS. TIBS is just emerging and is subject to repeated study from other groups for further evaluation.

\section{Prospective Medical Application of TIBS With a Targeted High-Z Prodrug}

In a clinical setting, ion beam energy is preferred, as it ensures accurate transmission to the target within the body. As proton energy modulates electron emission from NP [24], optimized energy is preferable to maximize dose enhancement at the target.

Because ion beam stimulation selectively activates NPs while saving NT, it is possible to treat multiple pathologic lesions that are mixed with or disseminated into NT, such as infiltrative

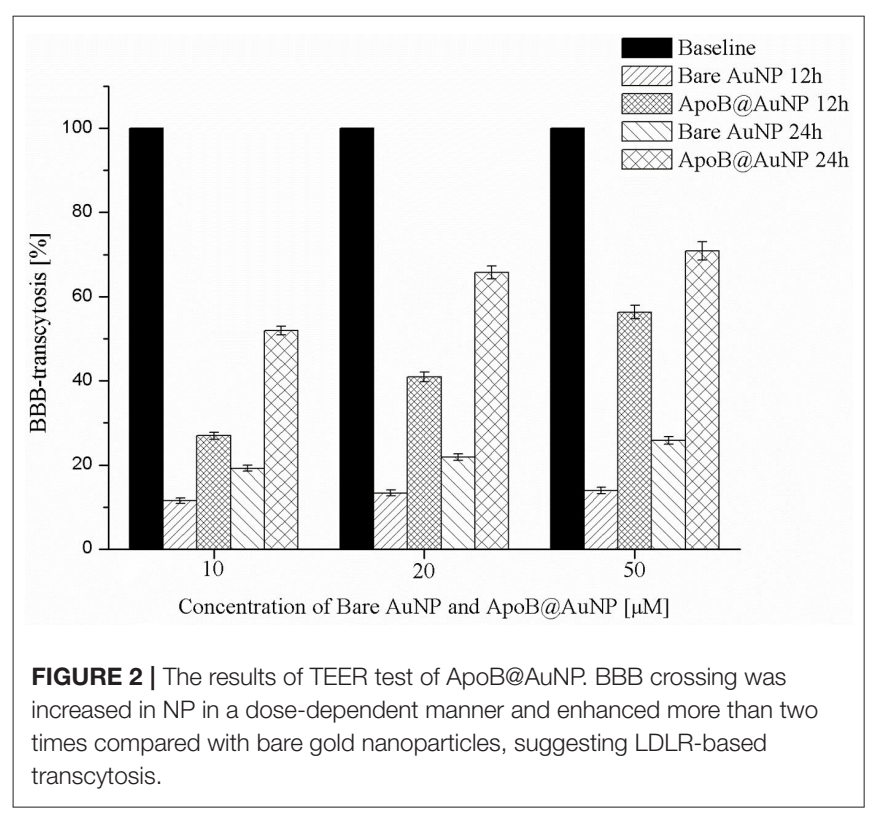

tumors, multiple cancer metastases, brain cancer in sensitive regions, vulnerable plaque or thrombosis in atherosclerotic blood vessels, neoangiogenesis-derived retinal diseases, and amyloid plaques in neurodegenerative diseases. All these pathologic hallmarks have previously been beyond the indications for conventional radiotherapy, including proton treatment, or surgical intervention. We demonstrated the feasibility of TIBS in animal models of these diseases with successful treatment in prior studies $[7,12,14,25]$ and with the proposed targeted NPs in the present study, as shown in Figures 2-4. In a tumor model $[7,12]$, we have shown that complete regression of smallsized tumors can be achieved by only proton-TIBS with a 510 Gy entrance dose under a given IV injection of iron oxide or gold NPs (100-300 mg/kg) without using BPD. Multiple small nodule-like lesions scattered in NT often have been encountered in various invasive cancers or in recurrences in adjacent critical function organs.

In a carotid arterial thrombus mouse model [14], we delivered $100 \mathrm{mg} / \mathrm{BW}$ iron oxide NPs via intravenous thrombus-derived obstruction in the affected carotid artery prior to single TIBS with a 2-4 Gy entrance dose.

Flow recovery was observed only in CNR-treated mice, with $>50 \%$ removal of the thrombus, without damaging the vascular endothelium. A 2.5-fold greater reduction in thrombus-enabled flow recovery was observed in the CNR group than in the protononly control groups $(p<0.01,14)$. In a transgenic AD mouse model [25], we submitted the results of the study to a separate journal for publication. Taken together, the results of the proofof-concept studies suggest the feasibility of TIBS for overcoming the challenges of treating infiltrative brain tumors, ruptured vulnerable plaques with thrombosis, or diabetic macular edema (DME)-associated retinal vein occlusion (RVO).

In an F98 infiltrative glioma rat model, we administered blood-brain barrier (BBB)-permeable and glioma-targeting LDLR-receptor-binding ApoB@AuNPs-Cy5 intravenously $24 \mathrm{~h}$ prior to proton-TIBS. The result of the TEER test demonstrated an enhanced efficiency in BBB crossing of APoB@AuNP compared with bare AuNP as shown in Figure 2. Red fluorescence of cyanine indicated delivery of NPs to both the
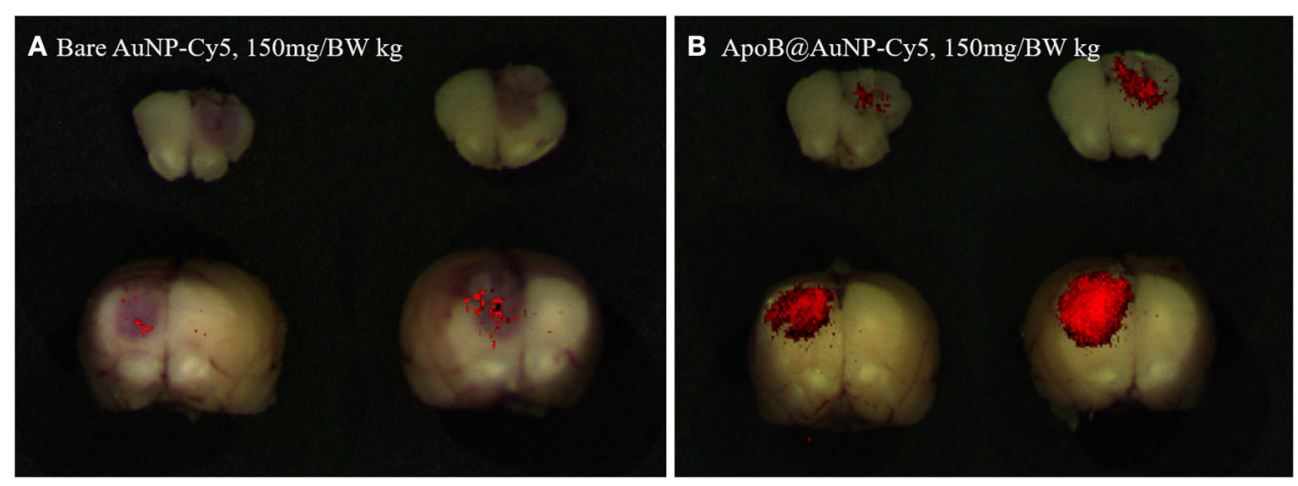

FIGURE 3 | Fluorescence imaging of AuNP-Cy5 (A) and ApoB@AuNP-Cy5 (B) that were taken up in glioma target at $24 \mathrm{~h}$ after IV injection of 150 mg/BW kg in glioma model. Much larger targeted-NP was taken up in the glioma mass compared with bare NP. 


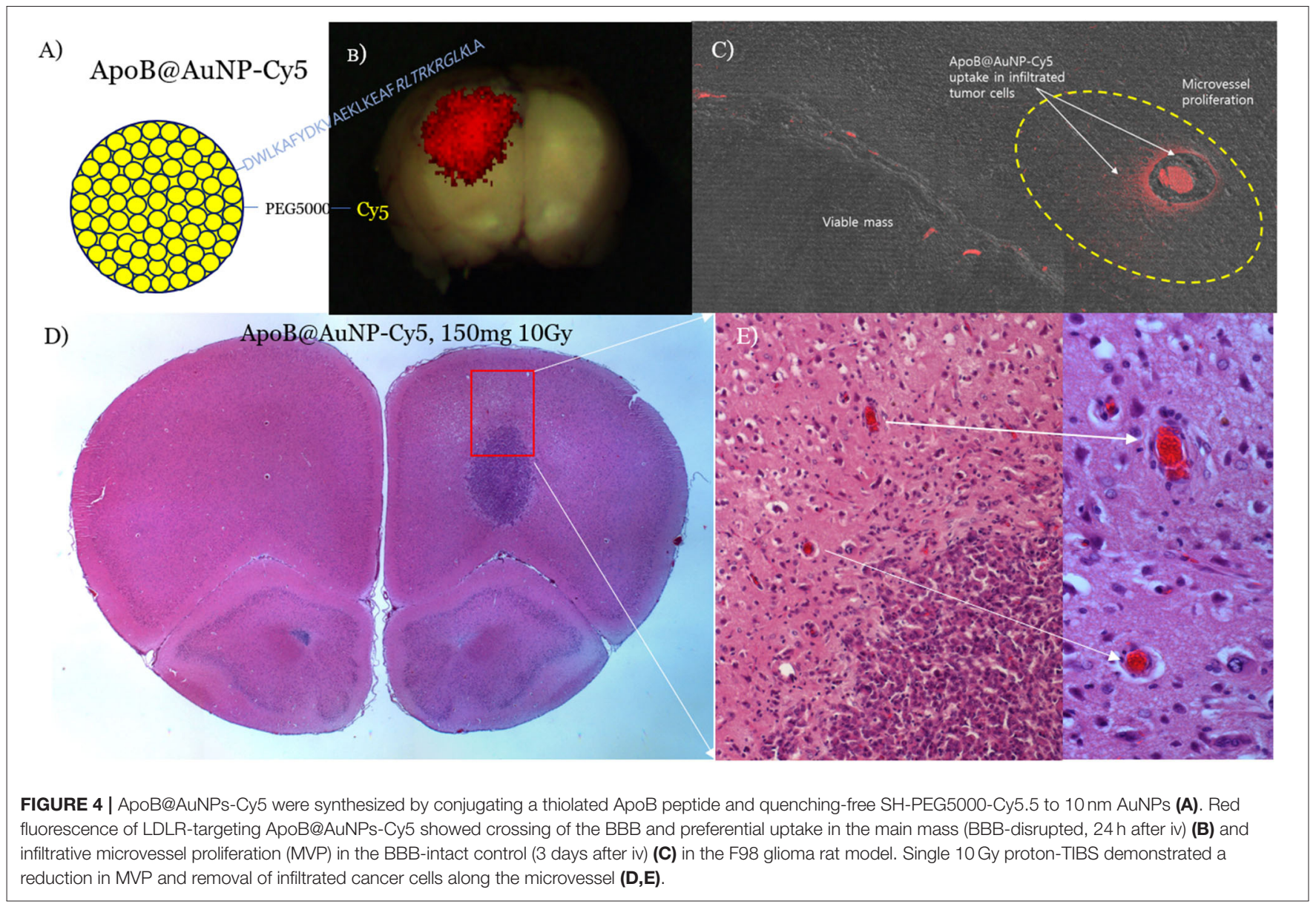

main mass and infiltrative microvessel proliferation (MVP), as shown in Figures 3, 4B,C, suggesting BBB crossing. Fluorescence imaging of molecular targeted ApoB@AuNPs-Cy5 demonstrated an uptake 11 times larger in the glioma target compared with non-targeted bare gold NP as shown in Figure 2. All rats presented appropriate MVP surrounding the glioma tumor mass. MVP was controlled in a dose-dependent manner; two were completely responsive (CR), and one had partial regression (PR) in 10 Gy-treated rats, in 5 Gy-treated rats, one was CR and two were PR. Proton-TIBS with an entrance dose of $10 \mathrm{~Gy}$ yielded an absence of cancer cells along the microvascular endothelium in Figures 4D,E. MVP was distributed in the surrounding $\mathrm{NT}$ where the $\mathrm{BBB}$ was intact in contrast to areas where the $\mathrm{BBB}$ was ruptured in the main tumor mass. Therefore, this result suggested that ApoB@AuNPs-Cy5 crossed the BBB since overexpression of LDLR was observed in both BBB and glioma cells.

Due to the fact that activated macrophages are taken up in vulnerable plaques of atherosclerotic blood vessels, scavenger receptor (SR-A1)-binding PP1-conjugated gold NPs (PP1@AuNPs) (Figure 5A) can be taken up by the plaque in a ligated-artery atheroma mouse model (Figure 5B). We achieved flow recovery from atheromatous obstruction of blood vessels in all three mice by reducing the plaque with 4-Gy protonTIBS, as demonstrated in Figures 4C,D. Recently, we found thrombolytic fucoidan that activated plasma tPA by binding with the tPA-inhibitor PAI-1, which induced thrombolysis in a thrombosis animal model [26]. We propose thrombus-targeting fucoidan-conjugated iron oxide NPs (TB-fuco@Fe3O4), as shown in Figure 6A, to treat DME with RVO or VEGFR-binding Flt1@AuNPs [10] to treat neoangiogenesis-derived age-related macular degeneration (AMD), or diabetic macular retinopathy, as shown in Figure 5. Because fucoidan is also known to bind selectively to P-selectin overexpressed in active thrombi [27], TB-fuco@Fe3O4 can be potentially targeted to sites of thrombosis in RVO prior to TIBS, which may induce flow recovery by a combined nanoradiator dose and thrombolytic fucoidan. We tested this hypothesis by TIBS after administration of TB-fuco@Fe3O4 in the same artery ligation atheroma model, where atheroma can often be accompanied by a thrombus, as shown in Figure 6B. Proton-TIBS with 4Gy achieved flow recovery by reducing both the thrombus and atheroma without damaging the normal architecture of the blood vessels, as shown in Figure 6C. We also developed VEGFR-binding Flt1@AuNPs-Cy5 that showed VEGFR binding and transcytosis in HRMECs, as shown in Figures 6D-F. Fluorescence imaging showed binding of Flt1@AuNPs-Cy5 to VEGFR in the membrane (Figure 6E) and subsequent transcytosis (Figure 6F). These NPs were proposed to treat AMD or DMR through the ocular DDS system, as reported previously [28], for the treatment 

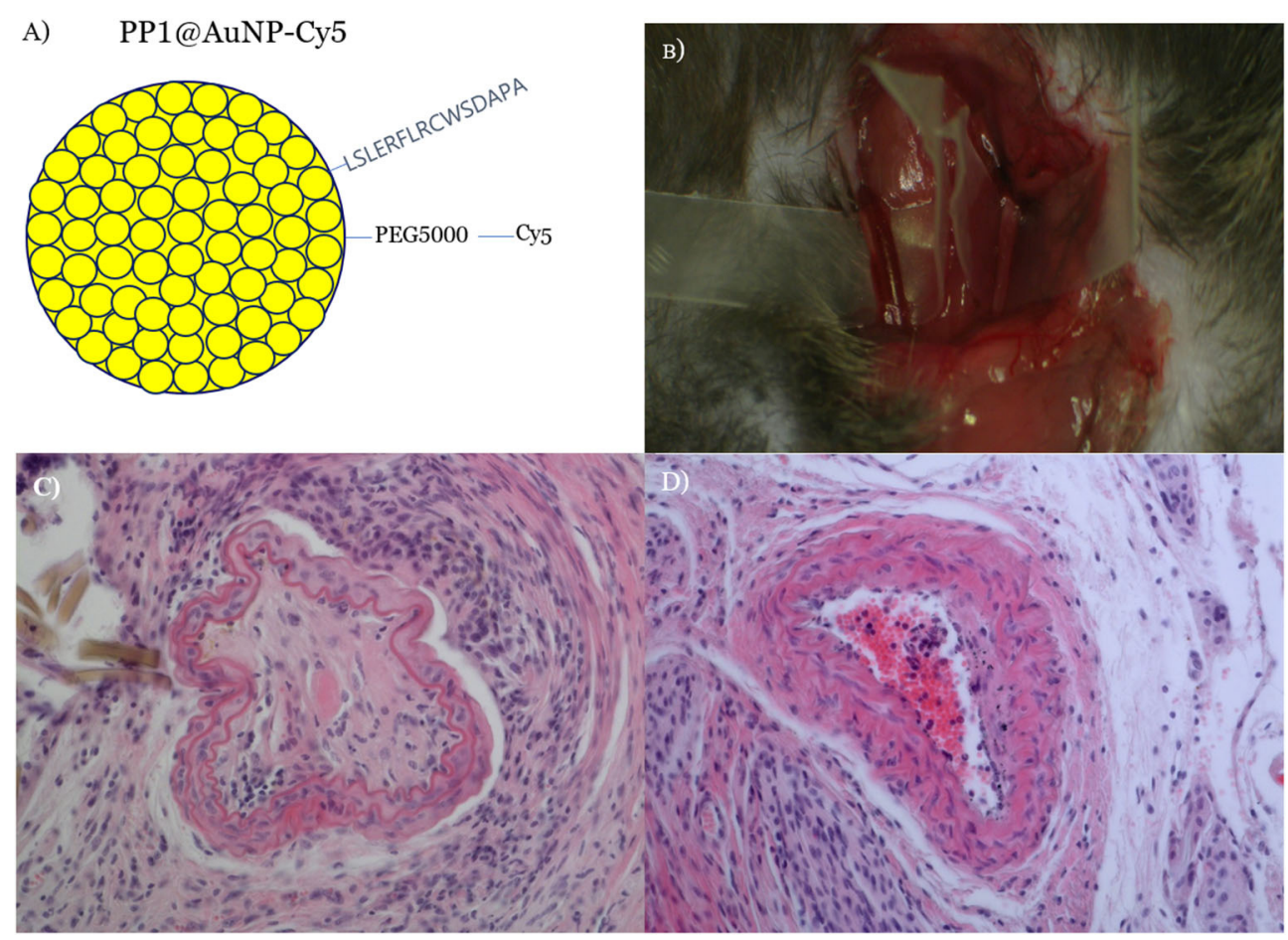

FIGURE 5 | SR-A1-binding PP1@AuNPs were prepared (A) and delivered via IV in a carotid artery-ligated atheromatous mouse model (B). Proton-TIBS (4 Gy) induced flow recovery by reducing plaque and preserving the normal structure of blood vessels (D) compared with the untreated obstruction control (C). The patency of the blood vessel was checked by a Doppler flow probe [21] and red blood cells were shown due to recovered blood flow in the central part of the treated blood vessel.

\section{A) Fuc@Fe3O4}

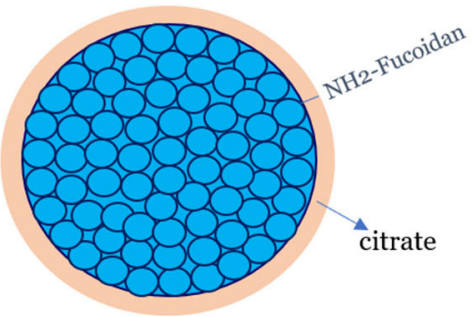

D) anti-Flt1@ AuNP-Cy5

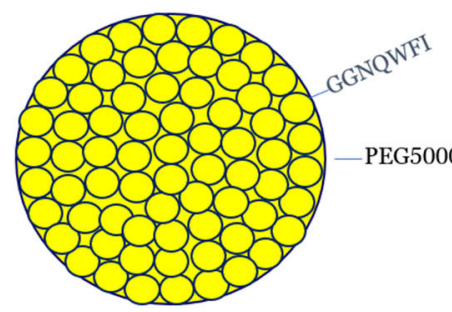

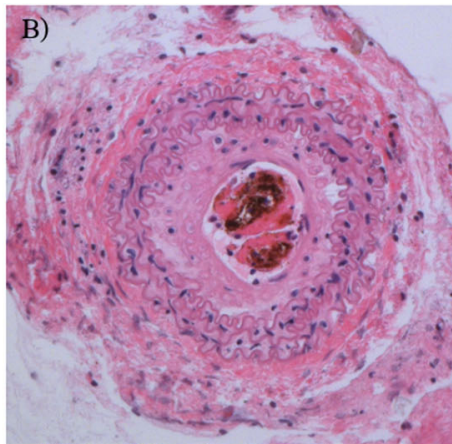
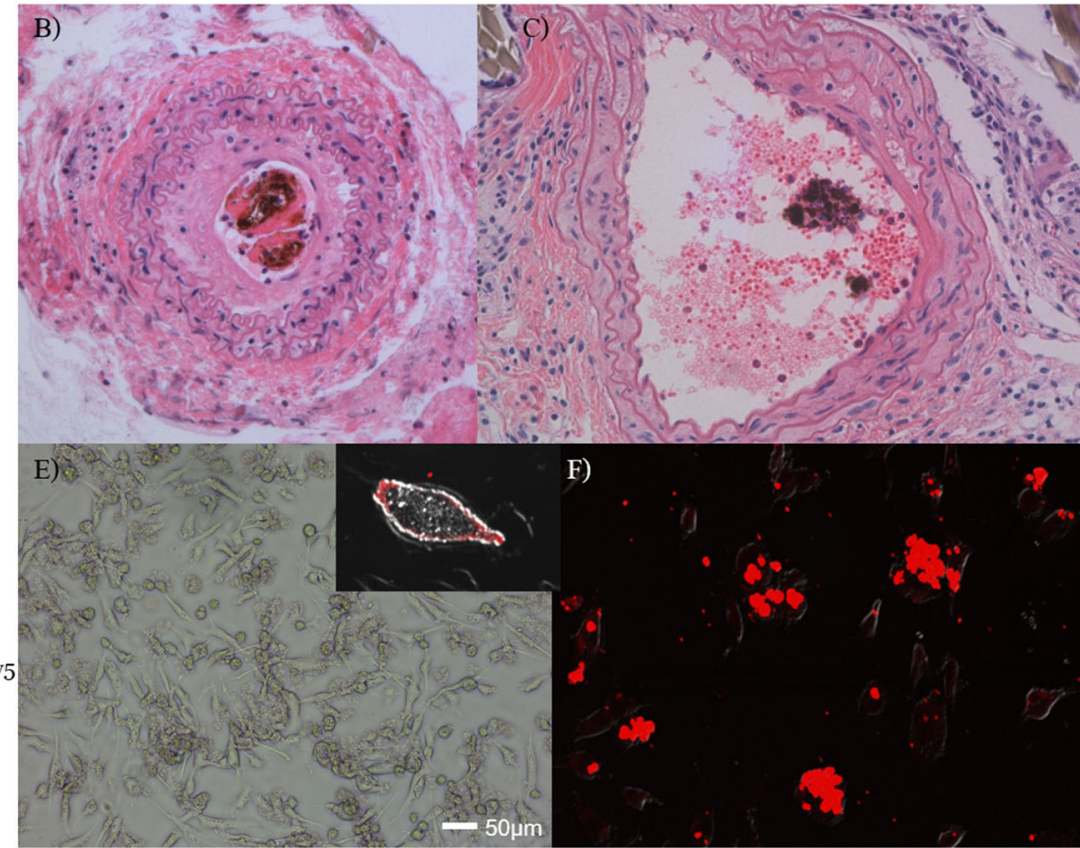

F)

FIGURE 6 | A thrombolytic aminated fucoidan, targeting active thrombi, was conjugated to citrated iron oxide NPs (A) and delivered to a carotid artery-ligated and lipid-fed atheromatous mouse model with accompanied thrombosis (B). Next, 4 Gy proton TIBS showed flow recovery and partial removal of both the central thrombus and atheroma $\mathbf{( C )}$. Dark materials in $\mathbf{( B , C )}$ were clusters of iron oxide NP that were taken up in thrombus. Central red blood cells in $\mathbf{( C )}$ indicated flow-recovery after treatment that was checked by a Doppler flow probe. VEGFR1(Flt1)-binding Flt1@AuNPs-Cy5 were prepared (D) and showed receptor binding in the membrane (E) and transcytosis in HRMECs (F) [21]. TIBS using either Fuc@Fe3O4 or Flt1@AuNPs will be applied to DME/RVO or AMD retinal disease models, respectively. 
of abnormal angiogenesis and endothelial cells together using TIBS [10].

Furthermore, TIBS can be easily implemented with highdose radiotherapy, such as FLASH or microbeam radiotherapy (MRT), which are known to have NT-preserving effects. This combination may greatly enhance therapeutic precision in addition to selective activation of targeted high- $\mathrm{Z}$ nanoparticles by TIBS. Overall, TIBS may provide new precision medicine through the development of targeted high-Z NPs; precision is achieved by nano-to-microscale transport of the CNR, with accompanying ROS generation and selective activation of NPs at the target site.

For further development of treatment planning, we aim to develop methodology to calculate the electron emissions from combined Auger cascades and an ICD path from high- $Z$ nanoparticles under TIBS. This may be put into the treatment planning of TIBS in a clinical setting.

\section{DATA AVAILABILITY STATEMENT}

The raw data supporting the conclusions of this article will be made available by the authors, without undue reservation.

\section{REFERENCES}

1. Newhauser WD, Zhang R. The physics of proton therapy. Phys Med Biol. (2015) 60:R155. doi: 10.1088/0031-9155/60/8/R155

2. Ulmer W, Matsinos E. Theoretical methods for the calculation of Bragg curves and 3D distributions of proton beams. Eur Phys J Spec Top. (2011) 190:1-81. doi: 10.1140/epjst/e2010-01335-7

3. Wälzlein C, Scifoni E, Krämer M, Durante M. Simulations of dose enhancement for heavy atom nanoparticles irradiated by protons. Phys Med Biol. (2014) 59:1441-58. doi: 10.1088/0031-9155/59/6/1441

4. Kim KH, Kim HT, Kim JH, Seo SJ, Chung DS, Kim JK. Investigation of tumor cell cytoxicity from particle induced x-ray emission from $45 \mathrm{MeV}$ proton beam irradiated ferrite nanoparticle. Int J PIXE. (2009) 19:14355. doi: 10.1142/S0129083509001837

5. Kim HK, Titze J, Schöffler M, Trinter F, Waitz M, Voigtsberger J, et al. Enhanced production of low energy electrons by alpha particle impact. Proc Natl Acad Sci USA. (2011) 108:11821. doi: 10.1073/pnas.1104 382108

6. Kim HK, Gassert H, Schoffler MS, Titze JN, Waitz M, Voigtsberger J, et al. Ion-impact-induced interatomic Coulombic decay in neon and argon dimers. Physical Rev A. (2013) 88:042707. doi: 10.1103/PhysRevA.88.042707

7. Kim JK, Seo SJ, Kim HT, Kim KH, Chung MH, Kim KR, et al. Enhanced proton treatment in mouse tumors through proton irradiated nanoradiator effects on metallic nanoparticles. Phys Med Biol. (2012) 57:8309. doi: 10.1088/0031-9155/57/24/8309

8. Barrios R, Skurski P, Simons J. Mechanism for damage to DNA by low-energy electrons. J Phys Chem B. (2002) 106:7991-4. doi: 10.1021/jp013861i

9. Alizadeh E, Orlando TM, Sanche L. Biomolecular damage induced by ionizing radiation: the direct and indirect effects of low-energy electrons on DNA. Annu Rev Phys Chem. (2015) 66:379-98. doi: 10.1146/annurev-physchem-040513-103605

10. Seo SJ, Lee SH, Kim KH, Kim JK. Anti-Flt1 peptide and cyanine-conjugated gold nanoparticles for the concurrent antiangiogenic and endothelial cell proton treatment. J Biomed Mater Res B Part B. (2019) 107:127283. doi: 10.1002/jbm.b.34220

11. Soeda S, Ohmagari Y, Shimeno H, Nagamatsu A. Preparation of aminated fucoidan and its evaluation as an antithrombotic and antilipemic agent. Biol Pharm Bull. (1994) 17:784-8. doi: 10.1248/bpb.17.784

\section{ETHICS STATEMENT}

The animal study was reviewed and approved by Institutional Animal Care and Use Committee of Catholic University Hospital of Daegu.

\section{AUTHOR CONTRIBUTIONS}

J-KK designed all the experiments and wrote the manuscript. S-JS carried out the experiments on carotid artery-ligated atheroma model with synthesizing molecular targeting gold nanoparticles. J-GJ carried out the experiment on infiltrative glioma model. All authors contributed to the article and approved the submitted version.

\section{FUNDING}

This work was partly supported by the Rare Isotope Science Project of the Institute for Basic Science funded by the Ministry of Science and ICT and NRF of Korea (2013M7A1A1075764).

12. Seo SJ, Jeon JK, Jeong EJ, Chang WS, Choi GH, Kim JK. Enhancement of tumor regression by coulomb nanoradiator effect in proton treatment of iron-oxide nanoparticle-loaded orthotopic rat glioma model: implication of novel particle induced radiation therapy. J Cancer Ther. (2013) 4:2532. doi: 10.4236/jct.2013.411A004

13. Nam D, Ni CW, Rezvan A, Suo J, Budzyn K, Llanos A, et al. Partial carotid ligation is a model of acutely induced disturbed flow, leading to rapid endothelial dysfunction and atherosclerosis. Am J Physiol Heart Circ Physiol. (2009) 297:H1535-43. doi: 10.1152/ajpheart.00510.2009

14. Jeon JK, Han SM, Min SK, Seo SJ, Ihm K, Chang WS, et al. Coulomb nanoradiator-mediated, site-specific thrombolytic proton treatment with a traversing pristine Bragg peak. Sci Rep. (2016) 6:37848. doi: 10.1038/srep37848

15. Arami $\mathrm{H}$, Khandhar $\mathrm{A}$, Liggitt $\mathrm{D}$, Krishnan $\mathrm{KM}$. In vivo delivery, pharmacokinetics, biodistribution and toxicity of iron oxide nanoparticles. Chem Soc Rev. (2015) 44:8576. doi: 10.1039/C5CS00541H

16. Carnovale C, Bryant G, Shukla R, Bansal V. Gold nanoparticle biodistribution and toxicity: role of biological corona in relation with nanoparticle characteristics. In: Rai M, Shegokar R, editors. Metal Nanoparticles in Pharma. Cham: Springer (2017). pp. 419-36.

17. Almeida JP, Chen AL, Foster A, Drezek R. In vivo biodistribution of nanoparticles. Nanomedicine. (2011) 6:815-35. doi: 10.2217/nnm.11.79

18. Mutlu H, Geiselhart CM, Barner-Kowollik C. Untapped potential for debonding on demand: the wonderful world of azo-compounds. Mater Horiz. (2018) 5:162-83. doi: 10.1039/C7MH00920H

19. Montenegro M, Nahar SN, Pradhan AK, Huang K, Yu, Y. Monte Carlo simulations and atomic calculations for auger processes in biomedical nanotheranostics. J Phys Chem A. (2009) 113:12364-9. doi: 10.1021/jp905323y

20. Seo SJ, Kim TJ, Hyodo K, Zaboronok A, You H, Peach K, et al. Enhanced generation of reactive oxygen species by the nanoradiator effect from coreinner shell photo-excitation or proton impact on nanoparticle atomic clusters. Radiat Environ Biophys. (2015) 54:423-31. doi: 10.1007/s00411-015-0612-7

21. Hainfeld JF, Slatkin DN, Smilowitz HM. The use of gold nanoparticles to enhance radiotherapy in mice. Phys Med Biol. (2004) 49:N30915. doi: 10.1088/0031-9155/49/18/N03

22. Jafari S, Cheki M, Tavakoli MB, Zarrabi A, Ghazikhanlu Sani K, Afzalipour R. Investigation of combination effect between $6 \mathrm{MV} \mathrm{X}$-ray radiation and polyglycerol coated superparamagnetic iron oxide nanoparticles on U87-MG cancer cells. J Biomed Phys Eng. (2020) 10:15-24. doi: 10.31661/jbpe.v0i0.929 
23. Choi GH, Seo SJ, Kim KH, Kim HT, Park SH, Lim JH, et al. Photon activated therapy (PAT) using monochromatic Synchrotron $\mathrm{x}$-rays and iron oxide nanoparticles in a mouse tumor model: feasibility study of PAT for the treatment of superficial malignancy. Radiat Oncol. (2012) 7:184. doi: 10.1186/1748-717X-7-184

24. Seo SJ, Jeon JK, Han SM, Kim JK. Reactive oxygen species-based measurement of the dependence of the Coulomb nanoradiator effect on proton energy and atomic $Z$ value. Int J Radiat Biol. (2017) 93:1239. doi: 10.1080/09553002.2017.1361556

25. Kim JK, Jeon JG, Chang WS, Choi YS. Proton stimulation targeting A $\beta$ plaque-associated magnetite reduces both iron redox toxicity and the plaque burden and improves cognitive function in an Alzheimer's disease mouse model (2020).

26. Choi YS, Min SK, Usoltseva R, Silchenko A, Zvyagintseva T, Ermakova S, et al. Thrombolytic fucoidans inhibit the tPA-PAI1 complex, indicating activation of plasma tissue-type plasminogen activator is a mechanism of fucoidanmediated thrombolysis in a mouse thrombosis model. Thromb Res. (2018) 161:22-5. doi: 10.1016/j.thromres.2017.11.015
27. Rouzet F, Bachelet-Violette L, Alsac JM, Suzuki M, Meulemans A, Louedec $\mathrm{L}$, et al. Radiolabeled fucoidan as a p-selectin targeting agent for in vivo imaging of platelet-rich thrombus and endothelial activation. J Nucl Med. (2011) 52:1433-40. doi: 10.2967/jnumed.110.085852

28. Wang C, Seo SJ, Kim JS, Lee SH, Jeon JK, Kim JW, et al. Intravitreal implantable magnetic micropump for on-demand VEGFR targeted drug delivery. J Control Rel. (2018) 283:105-12. doi: 10.1016/j.jconrel.2018.05.030

Conflict of Interest: The authors declare that the research was conducted in the absence of any commercial or financial relationships that could be construed as a potential conflict of interest.

Copyright (c) $2020 \mathrm{Kim}$, Seo and Jeon. This is an open-access article distributed under the terms of the Creative Commons Attribution License (CC BY). The use, distribution or reproduction in other forums is permitted, provided the original author(s) and the copyright owner(s) are credited and that the original publication in this journal is cited, in accordance with accepted academic practice. No use, distribution or reproduction is permitted which does not comply with these terms. 